\title{
Biomarkers and genetic variants reflect disease course in multiple sclerosis
}

\section{C4}

these findings

point towards

genetic and

molecular

pathways that

operate in

different types

of MS

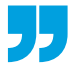

The pathogenesis of multiple sclerosis (MS) is known to encompass both inflammatory demyelination and neurodegeneration, but the factors that underlie these different aspects of the disease process are poorly understood. Two new studies have identified cerebrospinal fluid (CSF) biomarkers and genetic variants that correlate with the disease course in MS. These findings could open up new avenues of investigation into the pathophysiology of MS.

"There is evidence that glial responses and axonal damage are both present from the early stages of

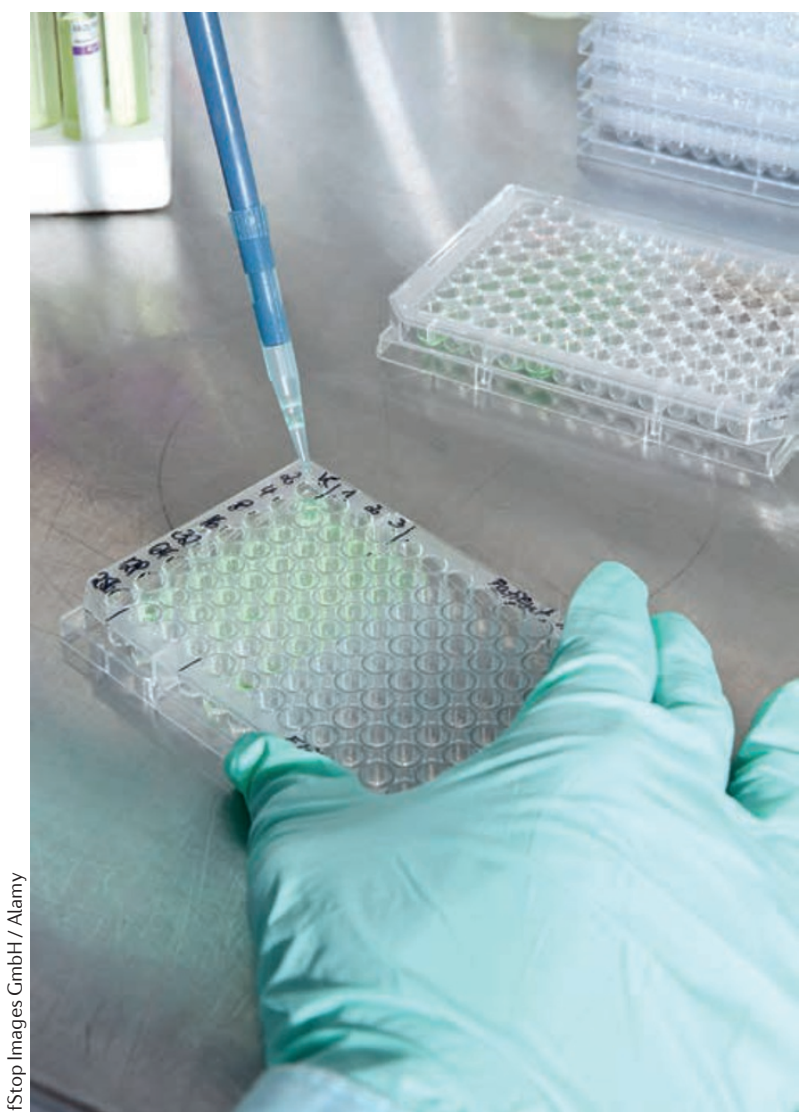

disease, and are important features in the pathogenesis of MS," says Maria Alba Mañé-Martínez, who led the CSF biomarker study. "The objectives of this research were to investigate glial and neuronal biomarkers in CSF samples from patients with different MS types, and to test whether a correlation among different biomarkers exists."

Mañé-Martínez and her colleagues collected CSF samples from 324 patients: 109 with clinically isolated syndrome (CIS), 192 with relapsingremitting MS (RRMS), six with secondary progressive MS, and 17 with primary progressive MS. The team used ELISA to measure levels of biomarkers that reflected various pathological processes, including axonal damage, neuronal injury, glial activation, astrocytic damage, and amyloid metabolism.

The researchers found that levels of neurofilament light protein (NFL), a marker of axonal damage, were elevated in the CSF of patients with RRMS. By contrast, increased concentrations of glial fibrillary acidic protein (GFAP), a marker of astrocytic damage, and YKL-40, a marker of glial activation, were associated with progressive forms of MS.

A subanalysis of the CIS and RRMS groups revealed differences in CSF biomarker profiles between the relapsing and remitting phases of MS. For example, levels of the glial activation marker MCP-1 (also known as CCL2) were increased during remission, whereas NFL concentrations seemed to peak towards the end of the relapse phase. Such fluctuations will need to be taken into account if these biomarkers are to be developed for use in the clinic.
In the second study, Bruce Taylor and colleagues set out to identify genetic variants that could predict the clinical course of MS. The researchers recruited 127 individuals who had recently experienced a first demyelinating event. The participants were genotyped for a panel of established MS-related single nucleotide polymorphisms (SNPs), and were followed up for 5 years.

Nine SNPs - two in HLA and seven in non-HLA regions of the genome - were found to predict conversion to MS and/or MS relapse. Interestingly, a completely different set of seven non-HLA SNPs was associated with disability progression, suggesting that relapsingremitting and progressive-onset diseases courses are determined by distinct genetic pathways.

Taken together, these findings point towards genetic and molecular pathways that operate in different types of MS and at different stages of the disease process. "If the present results could be confirmed in different cohorts of MS patients, some CSF biomarkers could be validated for clinical applicability," comments Mañé-Martínez. "It would also be interesting to investigate whether some of these biomarkers could be used to differentiate myelitis and optic neuritis from the early stages of MS."

Heather Wood

ORIGINAL ARTICLES Mañé-Martínez, M. A. et al. Glial and neuronal markers in cerebrospinal fluid in different types of multiple sclerosis.

J. Neuroimmunol.http://dx.doi.org/10.1016/ j.jneuroim.2016.08.004 (2016) | Pan, G. et al. Role of genetic susceptibility variants in predicting clinical course in multiple sclerosis. J. Neurol. Neurosurg. Psychiatry http://dx.doi.org/10.1136/ jnnp-2016-313722 (2016) 